05

\title{
Динамика самопроизвольных потерь намагниченности магнитов
} (Pr,Dy)-(Fe,Co)-B

\author{
(С) И.И. Резчикова, ${ }^{1}$ Н.С. Моисеева, ${ }^{1}$ Д.В. Королев, ${ }^{1}$ Р.Б. Моргунов, ${ }^{1,2}$ В.П. Пискорский ${ }^{1}$ \\ ${ }^{1}$ Всероссийский институт авиационных материалов, \\ 105005 Москва, Россия \\ ${ }^{2}$ Институт проблем химической физики РАН, \\ 142432 Черноголовка, Московская обл., Россия \\ e-mail: rezchikovaii@mail.ru
}

Поступило в Редакцию 3 апреля 2019 г.

В окончательной редакции 8 сентября 2019 г.

Принято к публикации 18 сентября 2019 г.

Сравнение самопроизвольных потерь намагниченности в спеченных магнитах $\left(\mathrm{Pr}_{0.51} \mathrm{Dy}_{0.49}\right)_{13}\left(\mathrm{Fe}_{0.64} \mathrm{Co}_{0.36}\right)_{80} \mathrm{~B}_{7} \quad$ и $\quad\left(\mathrm{Pr}_{0.51} \mathrm{Dy}_{0.49}\right)_{13}\left(\mathrm{Fe}_{0.64} \mathrm{Co}_{0.36}\right)_{79} \mathrm{Cu}_{1} \mathrm{~B}$ at.\% показало, что легирование медью приводит к уменьшению этих потерь. Это связано как с фазовой гомогенизацией, уменьшающей поля рассеяния на границах фаз, так и с ростом среднего поля анизотропии и коэрцитивной силы, стабилизирующей временные характеристики магнита. Обнаружено, что при комнатной температуре динамика размагничивания описывается экспоненциальной функцией.

Ключевые слова: намагниченность, спеченные магниты, потери намагниченности, магнитная релаксация, размагничивающий фактор.

DOI: 10.21883/JTF.2020.03.48922.145-19

\section{Введение}

Использование магнитов в точных приборах (например, в навигационных устройствах) требует предвидения их поведения на десятки лет вперед при различных температурах, поэтому его временная стабильность (стойкость) должна быть известна заранее. Стойкость магнита зависит от его геометрии, потому что с увеличением размагничивающего фактора и размагничивающего поля необратимые потери намагниченности $\Delta \Psi$ также увеличиваются. Хорошо известно, что необратимые потери намагниченности определяются рабочей точкой магнита $[1,2]$. Например, рабочая точка $\left(P_{c}\right)$ магнита в форме прямоугольной призмы дается выражением [3]:

$$
P_{c}=1.77 \frac{h}{l w}[h(l+w)+l w]^{1 / 2} .
$$

Здесь $h$ - размер магнита по текстуре, $l$ - длина магнита, $w$ - ширина магнита.

В работе [4] приводятся данные по необратимым потерям намагниченности до $10^{4} \mathrm{~h}$. Подобное длительное время (более года) продолжались эксперименты в работе [5]. В работе [6] время эксперимента также доходило до $10^{3} \mathrm{~h}$, но экстраполяцию делали до $10^{5} \mathrm{~h}$ (почти 10 лет). В работе [7] время эксперимента составляло примерно $10^{3} \mathrm{~h}$, а экстраполяция была до $10^{6} \mathrm{~h}$. Измерения потокосцепления в этих опытах, как правило, производят в катушках Гельмгольца [4-7]. Зависимость $\Delta \Psi(t)$ легко экстраполируется, поскольку обычно она подчиняется логарифмическому закону от времени выдержки $[8,9]$. Поэтому в полулогарифмических координатах имеет место легко экстраполируемая линейная зависимость $\Delta \Psi(\lg t)$. В литературе исследовали самопроизвольное размагничивание в $\mathrm{Sm}-\mathrm{Co}[10]$. В работах $[11,12]$ рассмотрена релаксация намагниченности материала $(\mathrm{Nd}, \mathrm{Sm}, \mathrm{Dy})-(\mathrm{Fe}, \mathrm{Co}) \mathrm{B}$. Зависимость релаксации намагниченности спрямляется в полулогарифмических координатах.

Целью настоящей работы является определение типа динамики необратимых потерь намагниченности в магнитах из спеченного материала $(\mathrm{Pr}, \mathrm{Dy})-(\mathrm{Fe}, \mathrm{Co})-\mathrm{B}$ прямоугольной формы при комнатной температуре.

Настоящая работа выполнена в рамках реализации комплексного научного направления 11.1: „Термостабильные магнитотвердые материалы и математические модели расчета их температурных характеристик для навигационных приборов нового поколения“ („Стратегические направления развития материалов и технологий их переработки на период до 2030 года“) [13].

\section{Материалы и методика эксперимента}

В наших опытах были исследованы спеченные материалы двух типов: $\mathrm{A} 1-\left(\operatorname{Pr}_{0.51} \mathrm{Dy}_{0.49}\right)_{13}\left(\mathrm{Fe}_{0.64} \mathrm{Co}_{0.36}\right)_{80} \mathrm{~B}_{7}$ и $\mathrm{A} 2-\left(\mathrm{Pr}_{0.51} \mathrm{Dy}_{0.49}\right)_{13}\left(\mathrm{Fe}_{0.64} \mathrm{Co}_{0.36}\right)_{79} \mathrm{Cu}_{1} \mathrm{~B}_{7}$. Здесь и далее составы даны в at.\%. В образцах составов $\quad\left(\operatorname{Pr}_{0.53} \mathrm{Dy}_{0.47}\right)_{13}\left(\mathrm{Fe}_{0.64} \mathrm{Co}_{0.36}\right)_{81} \mathrm{~B}_{6} \quad$ и $\left(\operatorname{Pr}_{0.53} \mathrm{Dy}_{0.47}\right)_{13}\left(\mathrm{Fe}_{0.64} \mathrm{Co}_{0.36}\right)_{80} \mathrm{Cu}_{1} \mathrm{~B}_{6}$ были определены содержание и состав фаз (табл. 1). Технология изготовления магнитов, методика исследований и фазовый состав описаны в работах $[14,15]$. Слитки дробили в конусной инерционной дробилке ВКМД 10 до размера менее $630 \mu \mathrm{m}$ в атмосфере азота. Дальнейшее 
Таблица 1. Результаты MPCA основных фаз в $\quad$ материалах состава $\left(\operatorname{Pr}_{0.53} \mathrm{Dy}_{0.47}\right)_{13}\left(\mathrm{Fe}_{0.64} \mathrm{Co}_{0.36}\right)_{81} \mathrm{~B}_{6}$ и $\left(\operatorname{Pr}_{0.53} \mathrm{Dy}_{0.47}\right)_{13}\left(\mathrm{Fe}_{0.64} \mathrm{Co}_{0.36}\right)_{80} \mathrm{Cu}_{1} \mathrm{~B}_{6}$ после отжига при температуре $500^{\circ} \mathrm{C}$

\begin{tabular}{c|c|c|c}
\hline Состав материала, at.\% & Фаза & Содержание, vol.\% & Состав фазы, at.\% \\
\hline \multirow{2}{*}{$\left(\operatorname{Pr}_{0.53} \mathrm{Dy}_{0.47}\right)_{13}\left(\mathrm{Fe}_{0.64} \mathrm{Co}_{0.36}\right)_{81} \mathrm{Ba}_{6}$} & $\mathrm{R}_{2} \mathrm{~F}_{14} \mathrm{~B}$ & \multirow{2}{*}{81.7} & $\left(\operatorname{Pr}_{0.51} \mathrm{Dy}_{0.49}\right)_{2}\left(\mathrm{Fe}_{0.66} \mathrm{Co}_{0.34}\right)_{14} \mathrm{~B}_{1}$ \\
\cline { 2 - 4 } & $\mathrm{R} 2 \mathrm{~F} 14 \mathrm{~B}$ & & $\left(\operatorname{Pr}_{0.61} \mathrm{Dy}_{0.39}\right)_{2}\left(\mathrm{Fe}_{0.66} \mathrm{Co}_{0.34}\right)_{14} \mathrm{~B}_{1}$ \\
\cline { 2 - 4 } & $\mathrm{RF}_{4} \mathrm{~B}$ & 14.8 & $\left(\operatorname{Pr}_{0.52} \mathrm{Dy}_{0.48}\right)_{1}\left(\mathrm{Fe}_{0.47} \mathrm{Co}_{0.53}\right)_{4} \mathrm{~B}_{1}$ \\
\cline { 2 - 4 } & $(\mathrm{R}, \mathrm{F})-\mathrm{O}$ & 1.8 & $\left(\operatorname{Pr}_{0.49} \mathrm{Dy}_{0.12} \mathrm{Fe}_{0.24} \mathrm{Co}_{0.15}\right)_{1} \mathrm{O}_{1}$ \\
\hline \multirow{2}{*}{$\left(\mathrm{Pr}_{0.53} \mathrm{Dy}_{0.47}\right)_{13}\left(\mathrm{Fe}_{0.64} \mathrm{Co}_{0.36}\right)_{80} \mathrm{Cu}_{1} \mathrm{~B}_{6}$} & $\mathrm{R}_{2} \mathrm{~F}_{14} \mathrm{~B}$ & 89.5 & $\left(\operatorname{Pr}_{0.51} \mathrm{Dy}_{0.49}\right)_{2}\left(\mathrm{Fe}_{0.64} \mathrm{Co}_{0.35} \mathrm{Cu}_{0.01}\right)_{14} \mathrm{~B}_{1}$ \\
\cline { 2 - 4 } & $\mathrm{RF}_{4} \mathrm{~B}$ & $\left(\operatorname{Pr}_{0.51} \mathrm{Dy}_{0.49}\right)_{1}\left(\mathrm{Fe}_{0.45} \mathrm{Co}_{0.53} \mathrm{Cu}_{0.02}\right)_{4} \mathrm{~B}_{1}$ \\
\cline { 2 - 4 } & $\mathrm{RF}$ & 6.6 & $\left(\operatorname{Pr}_{0.97} \mathrm{Dy}_{0.03}\right)\left(\mathrm{Fe}_{0.05} \mathrm{Co}_{0.07} \mathrm{Cu}_{0.88}\right)_{2}$ \\
\cline { 2 - 4 } & $(\mathrm{R}, \mathrm{F})-\mathrm{O}$ & \multirow{2}{*}{2.2} & $\left(\operatorname{Pr}_{0.69} \mathrm{Dy}_{0.25} \mathrm{Fe}_{0.03} \mathrm{Co}_{0.02} \mathrm{Cu}_{0.01}\right) \mathrm{O}_{2}$
\end{tabular}

Таблица 2. Размеры образцов (последний размер по текстуре), составы, плотность, свойства и величина рабочей точки магнитов

\begin{tabular}{c|c|c|c|c|c|c}
\hline № & Размеры образцов, $\mathrm{mm}$ & Состав & $p, \mathrm{~g} / \mathrm{cm}^{3}$ & $B_{r}, \mathrm{~T}$ & $H_{c i}, \mathrm{kA} / \mathrm{m}$ & $P_{c}$ \\
\hline P315-2 & $17.55 \times 9.8 \times 8.9$ & A1 & 7.87 & 0.889 & 1243 & 1.87 \\
P316-1 & $17.6 \times 10.95 \times 9.15$ & A2 & 7.83 & 0.839 & 1387 & 1.79
\end{tabular}

Примечание: $\mathrm{A} 1-\left(\operatorname{Pr}_{0.51} \mathrm{Dy}_{0.49}\right)_{13}\left(\mathrm{Fe}_{0.64} \mathrm{Co}_{0.36}\right)_{80} \mathrm{~B}_{7} ; \mathrm{A} 2-\left(\mathrm{Pr}_{0.51} \mathrm{Dy}_{0.49}\right)_{13}\left(\mathrm{Fe}_{0.64} \mathrm{Co}_{0.36}\right)_{79} \mathrm{Cu}_{1} \mathrm{~B}_{7}$.

измельчение проводили на центробежно-планетарной мельнице САНД-1 в среде SOLKANE $141 \mathrm{~b}$ CN (формула $\mathrm{C}_{2} \mathrm{FCl}_{2} \mathrm{H}_{3}$, температура кипения $31.9^{\circ} \mathrm{C}$ ) до среднего размера $10 \mu \mathrm{m}$. Заготовки магнитов в виде прямоугольных призм компактировали методом влажного прессования в магнитном поле $800 \mathrm{kA} / \mathrm{m}$, перпендикулярном направлению прессования. Спекание проводили в вакуумной печи СНВЭ-1.3.1/16-И3 при температуре $1120^{\circ} \mathrm{C}$ в течение $1 \mathrm{~h}$. Плотность определяли взвешиванием образцов в дистиллированной воде и на воздухе. После спекания образцы шлифовали алмазным инструментом в форме прямоугольных призм. Намагничивание проводили в импульсном поле величиной $1600 \mathrm{kA} / \mathrm{m}$. Магнитные измерения проводили на установке PERMAGRAPH C300 при комнатной температуре. Величину необратимых потерь намагниченности определяли измерением потокосцепления $\Psi$ в катушках Гельмгольца с помощью флюксметра EF5 при комнатной температуре путем сравнения $\Psi$ до и после выдержки образцов в термостате при заданной температуре. Магниты выдерживали в термостате при температуре $20^{\circ} \mathrm{C}$ в течение времени $1-3000 \mathrm{~h}$. Точность поддержания температуры составляла $\pm 2{ }^{\circ} \mathrm{C}$. Величину необратимых потерь намагниченности находили по формуле

$$
\Delta \Psi=(\Psi-\Psi(t)) / \Psi 100 .
$$

Здесь $\Psi(t)$ и $\Psi$ величины потокосцепления магнита в катушке Гельмгольца после выдержки в течение времени $t$ и потокосцепления образца в начальный момент време- ни соответственно. Величину рабочей точки магнитов вычисляли по формуле (1).

\section{Экспериментальные результаты}

Свойства магнитов представлены в табл. 2. Зависимость необратимых потерь намагниченности образца А1 от времени выдержки представлена на рис. 1. С целью определения закона релаксации намагниченности были подобраны параметры аппроксимирующих функций, представленных в работе [16]:

$$
\begin{gathered}
M(t)=M_{0} e^{(-t / \tau)}, \\
M(t)=M_{0}-S(H, T) \ln \left(\frac{t}{\tau}\right), \\
M(t)=M_{0}-S(H, T) \ln \left(1+\frac{t}{\tau}\right), \\
M(t)=M_{0} t^{-\xi}, \quad \xi<1, \\
M(t)=M_{0} e^{\left(-\frac{t}{\tau}\right)^{\beta}}, \quad \beta<1, \\
M(t)=M_{0} e^{\left(-\frac{t}{\tau}\right)^{\beta}}, \quad 1<\beta<3,
\end{gathered}
$$

где $\tau$ - время релаксации.

Экспоненциальный закон (дебаевская релаксация), описываемый функцией вида (3), применим для одной частицы или пленок с одним узким энергетическим барьером нуклеации или смещения доменных границ.

Функция вида (4a) описывает логарифмический закон для магнитных пленок с плоскостной анизотропией, 
(4б) - для пленок с перпендикулярной анизотропией. Временна́я зависимость намагниченности может быть описана логарифмическим законом при широком распределении высот энергетических барьеров.

Силовой закон релаксации описывается функцией вида (5). Такой тип наблюдали в спиновых стеклах и однодоменных ферромагнитных наночастицах.

Растянутая экспоненциальная релаксация, описываемая функцией вида (6), наблюдалась в пленках с логнормальным распределением энергетического барьера, в таких как разнообразные кластеры, аморфные материалы, сильно разупорядоченные пленки и спиновые стекла.

Сжатая экспоненциальная релаксация, описываемая функцией вида (7), применима для описания процессов размагничивания в спиновых стеклах, тонких пленках и многослойных образцах.

Из рис. 1 видно, что наилучшей аппроксимирующей функцией является функция вида (7) (релаксация Колмогорова-Аврами-Фатуззо), так как среднеквадратичное отклонение (СКО) наиболее близко к единице [16]. Для тонких пленок размагничивание является результатом разворота спинов, а также примыкающих к ним спинов за счет энергии обмена малой дальности, что представляет собой механизм зарождения доменов обратной намагниченности или нуклеацию. Такая модель описывает релаксацию, когда размагничивание управляется процессом нуклеации и ускоряется за счет движения доменных стенок [16].

На рис. 2 представлены зависимости необратимых потерь намагниченности, а также аппроксимации их

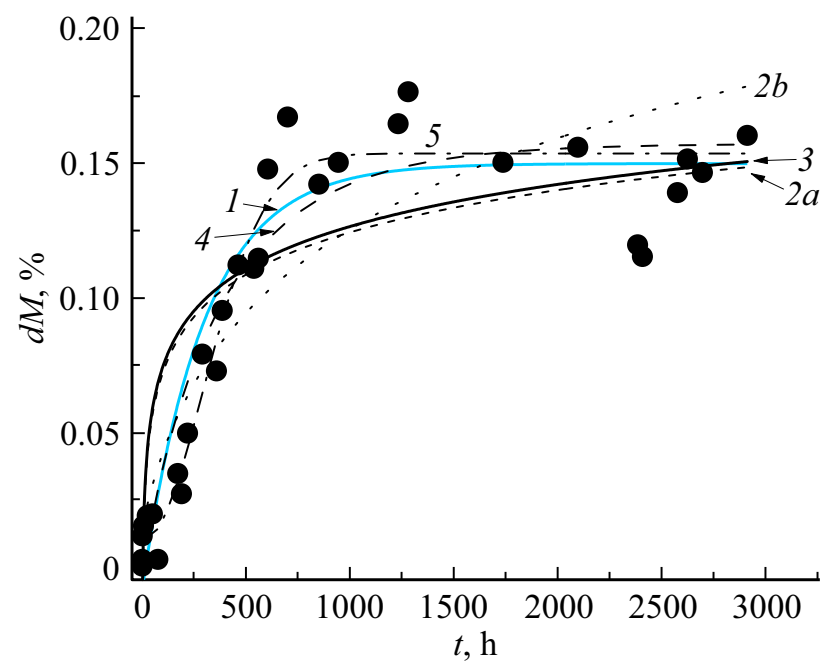

Рис. 1. Зависимость необратимых потерь намагниченности спеченного магнита состава $\left(\mathrm{Pr}_{0.51} \mathrm{Dy}_{0.49}\right)_{13}\left(\mathrm{Fe}_{0.64} \mathrm{Co}_{0.36}\right)_{80} \mathrm{~B}_{7}$ от времени выдержки при температуре $20^{\circ} \mathrm{C} ; 1$ - аппроксимация данных уравнением (3), СКО $=0.87 ; 2 a-$ аппроксимация данных уравнением (4a), $\mathrm{CKO}=0.67 ; 2 b-$ аппроксимация данных уравнением (4б), СКО $=0.77 ; 3-$ аппроксимация данных уравнением (5), СКО $=0.68 ; 4-$ аппроксимация данных уравнением (6), СКО $=0.87 ; 5-$ аппроксимация данных уравнением (7), СКО $=0.92$. Круглые метки - экспериментальные точки.

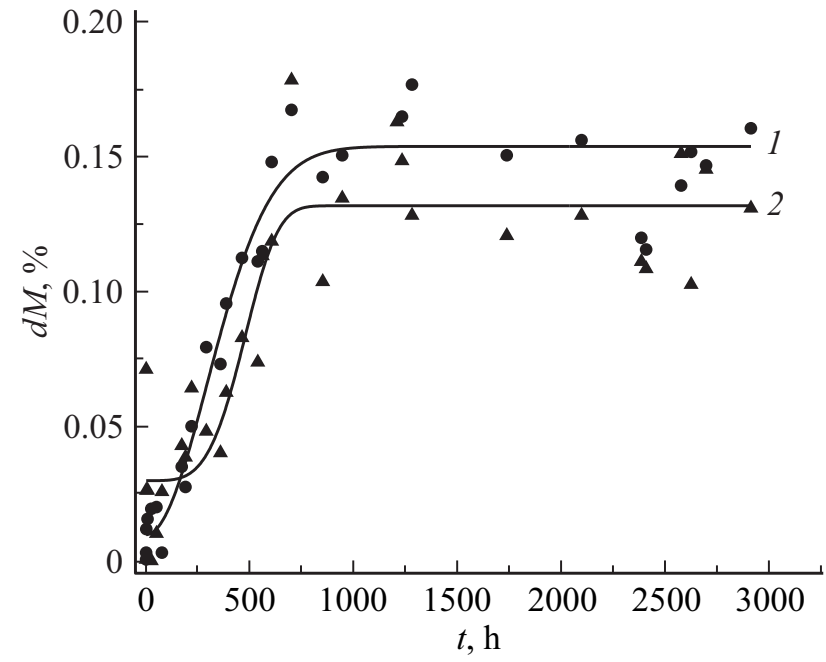

Рис. 2. Зависимость необратимых потерь намагниченности спеченных магнитов составов $\quad\left(\operatorname{Pr}_{0.51} \mathrm{Dy}_{0.49}\right)_{13}\left(\mathrm{Fe}_{0.64} \mathrm{Co}_{0.36}\right)_{80} \mathrm{~B}_{7}$ $\left(\mathrm{Pr}_{0.51} \mathrm{Dy}_{0.49}\right)_{13}\left(\mathrm{Fe}_{0.64} \mathrm{Co}_{0.36}\right)_{79} \mathrm{Cu}_{1} \mathrm{~B}_{7}$ (2) от времени выдержки при температуре $20^{\circ} \mathrm{C}$; - аппроксимация данных уравнением (8); $\mathbf{\Delta}$ - аппроксимация данных уравнением (9).

функцией (7) для образцов двух составов. Для образца состава А1 получена зависимость

$$
\Delta \Psi=0.15-0.14 e^{\left(-\frac{t}{420}\right)^{1.98}},
$$

для образца состава А2 получена зависимость

$$
\Delta \Psi=0.13-0.11 e^{\left(-\frac{t}{499}\right)^{2.9}} .
$$

Таким образом, время релаксации образца состава A1 составляет $420 \mathrm{~h}$, параметр $\beta$ равен 1.98 , что соответствует незначительному вкладу смещения доменных границ. Время релаксации образца состава А2 составляет $499 \mathrm{~h}$, параметр $\beta$ равен 2.9 , что свидетельствует о том, что вклад смещения доменных границ является доминирующим [16].

Из рис. 2 видно, что потери намагниченности образца, легированного медью, меньше по сравнению с образцом, в котором меди нет.

\section{Обсуждение результатов}

Известно, что величина необратимых потерь увеличивается с уменьшением значения рабочей точки магнита [4]. При одинаковых значениях $P_{c}$, величина $|\Delta \Psi|$ уменьшается с ростом коэрцитивной силы магнита $H H[4,9]$. Следует также отметить, что неоднородности внутренней структуры магнита также усиливают необратимые потери [9], поскольку, например, на границе раздела фаз $(\mathrm{Pr}, \mathrm{Dy})_{2}(\mathrm{Fe}, \mathrm{Co})_{14} \mathrm{~B}$ и $(\mathrm{Pr}, \mathrm{Dy})(\mathrm{Fe}, \mathrm{Co})_{4} \mathrm{~B}$ возникают домены обратной намагниченности в фазе 2-14-1, увеличивающие потери $|\Delta \Psi|$. Легирование спеченного материала $(\mathrm{Pr}, \mathrm{Dy})-(\mathrm{Fe}, \mathrm{Co})-\mathrm{B}$ 
медью приводит к уменьшению содержания в нем фазы $(\mathrm{Pr}, \mathrm{Dy})(\mathrm{Fe}, \mathrm{Co})_{4} \mathrm{~B}[17,18]$, т.е. к увеличению его однородности и соответствующему уменьшению величины потерь $\Delta \Psi$. Таким образом, причина увеличения потерь в магнитах состава A1 заключается в увеличенном количестве в них фазы $(\mathrm{Pr}, \mathrm{Dy})(\mathrm{Fe}, \mathrm{Co})_{4} \mathrm{~B}$. При этом видно, что у образца, легированного медью, коэрцитивная сила оказывается выше, чем в нелегированном образце. Это можно объяснить на основе данных табл. 1, где приведены результаты микрорентгеноспектрального анализа (МРСА) образцов, близких по составу к образцам $\left(\mathrm{Pr}_{0.51} \mathrm{Dy}_{0.49}\right)_{13.6}\left(\mathrm{Fe}_{0.64} \mathrm{Co}_{0.36}\right)_{79.6} \mathrm{~B}_{6.8}$ и $\left(\operatorname{Pr}_{0.51} \mathrm{Dy}_{0.49}\right)_{13.4}\left(\mathrm{Fe}_{0.64} \mathrm{Co}_{0.36}\right)_{78.8} \mathrm{Cu}_{1.1} \mathrm{~B}_{6.7}$. Видно, что при легировании медью происходит исчезновение фазы 2-14-1 с пониженным содержанием диспрозия. В то же время известно [19], что соединение $\mathrm{Dy}_{2} \mathrm{Fe}_{14} \mathrm{~B}$ обладает более высоким значением поля анизотропии $(12000 \mathrm{kA} / \mathrm{m})$ по сравнению с полем анизотропии соединения $\operatorname{Pr}_{2} \mathrm{Fe}_{14} \mathrm{~B}(600 \mathrm{kA} / \mathrm{m})$. Поэтому исчезновение фазы, обогащенной Pr, за счет увеличения доли фазы, обогащенной Dy, ведет к росту среднего значения магнитной анизотропии и соответствующему росту коэрцитивной силы. Таким образом, можно назвать, по крайней мере, два фактора, которые приводят к улучшению временной стабильности магнитов при легировании медью: фазовая гомогенизация и устранение фазы с низкой коэрцитивностью.

Обсудим теперь динамику размагничивания. Для обоих исследованных в настоящей работе сплавов наблюдается следующая закономерность: при комнатной температуре зависимость магнитных потерь от времени описывается функцией (7). Важно отметить, что ни при каких условиях динамика размагничивания не подчинялась логарифмической зависимости, обычно наблюдаемой в редкоземельных магнитах. Из этого можно сделать следующие предположения. Отсутствие логарифмической зависимости размагничивания от времени и наличие экспоненциальной зависимости говорит о том, что спектр времен релаксации намагниченности далек от равнораспределения и близок к ситуации, когда высота потенциальных барьеров релаксации одинакова в разных участках образца. Это может быть обусловлено равномерностью магнитной структуры и сильным взаимодействием между элементами спеченного магнита (зернами, фазами). Обычно такие зависимости магнитной релаксации от времени возникают в спиновых стеклах и материалах с непрерывным спектром времен релаксации, далеким от прямоугольного (который приводит к логарифмической зависимости).

\section{Выводы}

Уменьшение намагниченности с течением времени у образцов состава $\left(\mathrm{Pr}_{0.51} \mathrm{Dy}_{0.49}\right)_{13}\left(\mathrm{Fe}_{0.64} \mathrm{Co}_{0.36}\right)_{80} \mathrm{~B}_{7}$ больше по сравнению с образцами состава $\left(\operatorname{Pr}_{0.51} \mathrm{Dy}_{0.49}\right)_{13}\left(\mathrm{Fe}_{0.64} \mathrm{Co}_{0.36}\right)_{79} \mathrm{Cu}_{1} \mathrm{~B}_{7}$, что объясняется меньшей однородностью материала, связанной с повышенным количеством фазы $(\mathrm{Pr}, \mathrm{Dy})(\mathrm{Fe}, \mathrm{Co})_{4} \mathrm{~B}$, a также меньшей величиной коэрцитивной силы, обусловленной наличием фазы 2-14-1 с пониженным содержанием диспрозия.

Временная зависимость намагниченности материалов при комнатной температуре описывается экспоненциальной функцией. Причиной необратимых потерь намагниченности магнита состава A1 $\left(\mathrm{Pr}_{0.51} \mathrm{Dy}_{0.49}\right)_{13}\left(\mathrm{Fe}_{0.64} \mathrm{Co}_{0.36}\right)_{80} \mathrm{~B}_{7}$, вероятнее всего, является зарождение зародышей фазы обратной намагниченности фазе 2-14-1 с последующим их ростом, необратимые потери намагниченности магнита состава $\mathrm{A} 2\left(\mathrm{Pr}_{0.51} \mathrm{Dy}_{0.49}\right)_{13}\left(\mathrm{Fe}_{0.64} \mathrm{Co}_{0.36}\right)_{79} \mathrm{Cu}_{1} \mathrm{~B}_{7}$ обеспечиваются смещением доменных границ.

\section{Финансирование работы}

Работа выполнена при поддержке Министерства промышленности и торговли Российской Федерации.

\section{Конфликт интересов}

Авторы заявляют, что у них нет конфликта интересов.

\section{Список литературы}

[1] Strnat K.J., Xiao Y., Mildrum H.F. // J. Appl. Phys. 1988. Vol. 64. N 10. P. 5522-5524.

[2] ГОСТ 19693-74. Материалы магнитные. Термины и определения. М.: Изд-во стандартов, 1986. 32 с.

[3] Haavisto M. // Doctoral thesis. Tampere: Tampere University of Technology, 2013. $86 \mathrm{p}$.

[4] Haavisto M., Paju M. // IEEE Tr. Magn. 2009. Vol. 45. N 12. P. 5277-5280.

[5] Haavisto M., Tuominen S., Kankaanpää H., Paju M. // IEEE Tr. Magn. 2010. Vol. 46. P. 3582-3584.

[6] Haavisto M., Paju M. // Proc. 20th International Workshop on Rare Earth and Future Permanent Magnets and Their Applications. Beld. 2010. P. 224-226.

[7] Haavisto M., Tuominen S., Kankaanpää H., Paju M. // IEEE Tr. Magn. 2011. Vol. 47. P. 170-174.

[8] Wohlfarth E.P. // J. Phys. F: Met. Phys. 1984. Vol. 14. P. L155L159.

[9] Миткевич А.В. Стабильность постоянных магнитов. Л.: Энергия, 1971. 129 с.

[10] Liu J., Vora P., Dent P., Walmer M., Chen C., Talnagi J., Wu S., Harmer M. // Proc. Space Nucl. Conf. Boston, 2007. P. 2036.

[11] Дмитриев А.И., Кучеряев В.В., Кунищына Е.И., Валеев Р.А., Моргунов Р.Б., Пискорский В.П., Оспенникова О.Г., Каблов Е.Н. // ФТТ. 2016. Т. 58. Вып. 8. С. 1530 1533.

[12] Дмитриев А.И., Таланцев А.Д., Куницына Е.И., Моргунов Р.Б., Пискорский В.П., Оспенникова О.Г., Каблов Е.Н. // ЖЭТФ. 2016. Т. 150. № 2(8). С. 350-355.

[13] Каблов Е.Н. // Авиационные материалы и технологии. 2015. № 1. C. 3-33.

DOI: $10.18577 / 2071-9140-2015-0-1-3-33$ 
[14] Каблов Е.Н., Оспенникова О.Г., Пискорский В.П., Резчикова И.И., Валеев Р.А., Давыдова Е.А. // Авиационные материалы и технологии. 2015. № S2. С. 5-10. DOI: $10.18577 / 2071-9140-2015-0-S 2-5-10$

[15] Каблов Е.Н., Оспенникова О.Г., Резчикова И.И., Пискорский В.П., Валеев Р.А., Королев Д.В. // Авиационные материалы и технологии. 2015. № S2. С. 24-29. DOI: 10.18577/2071-9140-2015-0-S2-24-29

[16] Morgunov R., Lu Y., Lavanat M., Fache T., Deveaux X., Migot S., Kolpak O., Talantsev A., Mangin S. // Phys. Rev. B. 2017. Vol. 96. P. 054421.

[17] Каблов Е.Н., Пискорский В.П., Валеев Р.А., Волков Н.В., Давыдова Е.А., Шайхутдинов К.А., Балаев Д.А., Семенов С.В. // Металлы. 2014. № 1. С. 65-69.

[18] Каблов Е.Н., Оспенникова О.Г., Чередниченко И.В., Резчикова И.И., Валеев Р.А., Пискорский В.П. // Авиационные материалы и технологии. 2015. № S2. C. 11-19. DOI: $10.18577 / 2071-9140-2015-0-S 2-11-19$

[19] Herbst J.F. // Rev. Mod. Phys. 1991. Vol. 63. N 4. P. 819-898. 